\title{
Zebrafish Models of Biliary Atresia and Other Infantile Cholestatic Diseases
}

\author{
Zenobia C. Cofer $\cdot$ Randolph P. Matthews
}

Published online: 14 March 2014

(c) Springer Science+Business Media New York 2014

\begin{abstract}
Zebrafish are a powerful tool for the study of diseases that appear during development. Over the past decade, several zebrafish models of diseases that affect the developing liver in infants have been generated, including models of the most common identifiable cause of neonatal cholestasis, biliary atresia (BA). While none of these models exactly duplicate the disorder, studies on the zebrafish models have uncovered potentially important features of BA such as an importance of epigenetic changes and an association with specific mutations. In addition, other models replicate other cholestatic disorders, and there are zebrafish models of other pediatric liver diseases as well. Continued efforts with these models, and future models that take advantage of emerging technologies, will uncover additional facets of disease pathogenesis and may identify novel, more effective therapies.
\end{abstract}

Keywords Zebrafish model - Biliary atresia $\cdot$ Neonatal cholestasis - Epigenetic changes - Cholestatic disorders . Disease pathogenesis

\section{Introduction}

Over the past decade, zebrafish have emerged as an intriguing model for the study of liver diseases that primarily affect children. As zebrafish are a developmental

Z. C. Cofer · R. P. Matthews $(\square)$

The Children's Hospital of Philadelphia Research Institute, Division of Gastroenterology, Hepatology and Nutrition, Department of Pediatrics, Perelman School of Medicine at the University of Pennsylvania, 902C Abramson Research Bldg, 3615 Civic Center Blvd, Philadelphia, PA 19104, USA

e-mail: matthews@email.chop.edu model, they would seem to be well suited to this role, as most of these diseases first appear in infancy, when the liver and bile ducts continue to develop, and thus are likely to have a developmental component. While the etiologies of many of these disorders are not known, the zebrafish models have allowed us to uncover mechanisms important in mediating the defects in the fish. This has in turn yielded insight into disease pathogenesis, and our continued efforts using the models described below should uncover further aspects of pathogenesis and may suggest novel treatments.

\section{Liver Disease in Children}

To date, the greatest advances in our understanding of pediatric liver disease using zebrafish have come from efforts to model cholestatic disease. Cholestasis is defined as poor bile flow and is most frequently caused by obstruction or malformation of the biliary tree. Cholestasis manifests as jaundice and is associated with poor growth and malabsorption of fat-soluble vitamins that most frequently leads to excess bleeding from vitamin $\mathrm{K}$ deficiency. Bile is necessary for normal digestion of fat and excretion of toxins, but is itself toxic to liver cells, as accumulation of bile components in the liver results in inflammation and fibrosis. Over time, this can lead to liver failure and cirrhosis, which untreated can lead to lifethreatening sequelae such as severe infection and profound bleeding.

Approximately 1 in 3,000 infants presents with cholestasis, and the list of potential causes for infantile cholestasis is exhaustive [1]. Broadly, the causes include infections, metabolic disorders, and genetic and/or developmental disorders. The most common identifiable cause of infantile cholestasis is biliary atresia (BA), an idiopathic 
disorder that affects $\sim 1 / 12,000$ infants [2]. BA is characterized by a fibro-inflammatory destruction of the extrahepatic biliary tree and is also associated with intrahepatic bile duct proliferation that is probably secondary to the obstruction. If left untreated, there is progressive biliary cirrhosis, resulting in profound malnutrition and other complications, leading to death. Ten to 20 percent of patients with BA have a syndromic form of the disease, with other anomalies, most commonly involving defects in left-right asymmetry such as polysplenia and intestinal malrotation [3, 4]. The only treatment for BA is a surgical procedure (the Kasai portoenterostomy) that is not particularly effective, as many children with BA eventually require liver transplantation [5]. Although there have been considerable efforts to better understand BA, identification of an etiology remains elusive, and thus studies in zebrafish may provide some insight into pathogenesis.

While recent studies of BA have demonstrated possible genetic susceptibility, and some syndromic BA patients may have an underlying genetic disorder, a unifying genetic cause of BA is unlikely. In contrast, several genetic causes of cholestatic disease have been characterized, such as Alagille syndrome, arthrogryposis-renal dysfunctioncholestasis (ARC) syndrome, and North American Indian childhood cirrhosis (NAIC). Of note, these disorders have all been modeled in zebrafish (see below). Patients with these disorders often have defects in other organs. In Alagille syndrome, there is a wide heterogeneity of findings, but many patients have heart, skeletal, eye, and/or vascular defects [6]. In ARC, there are characteristic kidney, joint, and skin findings, as well as the liver defects [7]. NAIC is quite rare, but is characterized by intrahepatic findings similar to BA without extrahepatic obstruction [8]. The zebrafish models of these rare disorders provide opportunities for studying pathogenic mechanisms in a model organism.

Other genetic disorders that affect the developing liver lead to developmental biliary anomalies and are associated with liver fibrosis, and are also associated with the formation of kidney cysts [9]. Like patients with syndromic BA, patients with the cystic kidney diseases may have defects in left-right asymmetry. These disorders have been described mostly in genes associated with cilia, and patients with these ciliopathies may have a variety of developmental defects affecting multiple organ systems, including the liver [10]. There are several zebrafish models with cystic kidneys [11, 12], but there has not yet been extensive examination of liver phenotypes in these models.

In addition to the cholestatic disorders that affect the developing liver, there are also several metabolic disorders that affect children. While many of these also affect other organs, often the primary derangement is manifest most clearly in the liver. Symptoms of these disorders vary widely, but the liver disease can present as cholestasis or as acute liver failure (ALF). In ALF, the metabolic derangement typically results in hepatocyte death, which leads to an inflammatory response and continued destruction, resulting in impairment of liver function. ALF in infants and children can progress rapidly, requiring emergent liver transplantation or even leading to death [13]. Treatment of these disorders in patients requires quickly addressing the cause, if possible. Thus, animal models of these disorders may yield important understanding of underlying mechanisms that could lead to more effective treatments.

There are of course multiple disorders that affect the liver in older children, but most of these disorders are also seen in adults. In addition to the many viral infections that affect both adults and children, conditions such as autoimmune hepatitis, Wilson's disease, alpha-1-antitrypsin deficiency, and non-alcoholic steatohepatitis (NASH) are seen in older children. In fact, NASH is now the most common chronic liver disease affecting US children [14]. Several zebrafish models of these disorders have been established, but as these disorders are not unique to children, they will be discussed elsewhere.

\section{Zebrafish Models of BA}

Ideally, a zebrafish model of BA would have defects in the extrahepatic biliary tree that resemble the fibroinflammatory destruction seen in patients and would also have intrahepatic bile duct proliferation, activation of inflammatory pathways such as interferon-gamma, fibrogenesis, and the potential to be associated with left-right asymmetry defects. The most frequently utilized mouse model of BA, derived by injection of a strain of rotavirus from rhesus macaque (RRV) into mouse pups [15], has many of these features, but is of course derived from an infectious model and thus may not be relevant to all forms of BA in patients. Zebrafish offer a more facile model system, in which the study of multiple mechanisms that lead to a BAlike phenotype may lead to uncovering more universal factors in BA pathogenesis. To date, no zebrafish model possesses all of the above features of BA (though neither does the mouse model), but the existing models that do share elements with the disease have yielded some insights into pathogenesis.

The first zebrafish model to address BA directly was the mutant duct-trip, in which there is a mutation in the gene encoding $S$-adenosylhomocysteine hydrolase (AHCY) [16]. While the liver of homozygous $a h c y^{-/}$larvae had several abnormalities, there was a striking absence of intrahepatic bile ducts and reduction in the size of the gallbladder [17•]. We found that inhibition of DNA methylation, either with the chemical inhibitor 
5-azacytidine or by injecting morpholinos directed against dnmtl (DNA methyltransferase I), led to an identical biliary phenotype. While this alone was not enough to draw a parallel to BA, analysis of gene expression changes uncovered an activation of interferon-gamma target genes [17•] and a decrease in promoter methylation of ifng family genes as well [18]. Others had shown that interferongamma was upregulated in patients with BA [19], and studies in the mouse BA model showed that activation of interferon-gamma was critical for development of the disease phenotype [20]. Thus, inhibition of DNA methylation in zebrafish led to defects in the biliary tree and activation of interferon-gamma, which could be considered similar to BA. We examined DNA methylation in samples from patients with BA, which demonstrated lower levels of DNA methylation in bile duct cells in the BA patients [17•], further supporting a connection between the disorder and this zebrafish model. In addition, this last finding provided a potentially novel mechanism by which BA could arise in patients, as DNA methylation can be elicited by viruses, toxins, environmental effects, and genetically. Thus, establishment of this zebrafish model of BA, while not exactly duplicating all of the features of BA in patients, did uncover novel features of the disorder that may yield clues to pathogenesis.

Similarly, studies of potential genetic influences on BA using zebrafish have yielded models that share some features with the disease, enhancing our understanding of pathogenesis. Rare patients with defined genetic disorders have developed BA, and thus defects in the causative genes may be at least partially leading to the disease. We identified a patient with Mowat-Wilson syndrome, which is typically characterized by neurological deficits, who also had BA. Mowat-Wilson syndrome is caused by a mutation in ZFHX1B, a transcription factor associated with SMAD signaling. Using morpholinos, we knocked down $z f h x l b$ in zebrafish, which led to developmental biliary defects [21]. While this certainly does not demonstrate a causative role for $Z F H X 1 B$ in BA, it does suggest a potential importance of this gene and the pathway in general in bile duct formation and thus a possible role in mediating pathogenesis of BA and other cholestatic diseases.

Several groups have examined potential genetic influences on $\mathrm{BA}$ in genomewide association studies, uncovering possible roles for $G P C 1, A D D 3$, and XPNPEP1 [22••, 23-25], among others. We utilized a morpholino antisense oligonucleotide-mediated knockdown of the glypican $g p c 1$ to examine the effect of inhibition of gpcl in biliary development, which demonstrated both extra- and intrahepatic biliary defects and an activation of Hedgehog signaling [22•*]. Examination of GPC1 in patient samples showed a loss of staining in all BA samples examined, as well as a decrease in staining in other causes of cholestasis
[22••]. Glypicans had been shown to potentially act as a "sink" for Hedgehog ligand [26], and others had shown activation of Hedgehog in patients with BA [27], so our findings supported a role for this signaling pathway in the pathogenesis of $\mathrm{BA}$ and suggested a possible connection between a loss of GPC1 and activation of Hedgehog. Hedgehog signaling is important in fibrogenesis as well as in developmental pathways and is intimately associated with primary cilia [10], which have also been implicated in BA [28] and other cholestatic disorders [10]. Importantly, we were able to reverse the defects elicited by $g p c l$ knockdown by inhibiting Hedgehog activity [22••], again supporting gpcl knockdown as a BA model and potentially identifying treatment strategies for patients.

While most work to date on BA in zebrafish has focused on the biliary tract, more recently developed tools may lead to further examination of the importance of hepatic stellate cells (HSCs) and fibrogenesis. Yin et al. developed a transgenic reporter line in which HSCs are visible, allowing these cells to be tracked during development. Moreover, these investigators determined that inhibition of pathways such as VEGF and retinoic acid receptor signaling decreased the number of HSCs, while inhibition of retinoid $\mathrm{X}$ receptor signaling increased the number of HSCs [29・0]. These studies show the potential utility of such a reporter line in further studies on the importance of HSCs in liver disease in general and may specifically be able to examine the importance of these cells in diseases such as BA as well.

As mentioned above, abnormalities in primary cilia have been noted in patients with BA. Because primary cilia play an important role in Hedgehog signaling and in the establishment of left-right asymmetry, the link between primary cilia and BA is intriguing. While primary cilia have not yet been identified in developing cholangiocytes in zebrafish, abnormalities in pathways linked to primary cilia such as Hedgehog signaling and glypicans do affect biliary development in zebrafish. These pathways, along with cilia, are strongly linked to cell polarity pathways [30-32], and numerous models with abnormalities in cell polarity demonstrate developmental biliary defects. A few years ago, Sakaguchi et al. [33] demonstrated that disruption of the endothelial cells in the developing zebrafish liver led to abnormal hepatocyte apicobasolateral polarity, which in turn led to defects in intrahepatic bile duct development. Subsequent work has shown that mutation of specific genes associated with cell polarity such as cldn15lb (claudin 15-like b) leads to intrahepatic biliary defects, further supporting an importance of the development of apicobasolateral cell polarity in biliary development [34•].

In addition to apicobasolateral cell polarity, planar cell polarity (PCP), which mediates polarity within the epithelial sheet and is orthogonal to apicobasolateral polarity, 
also appears important in bile duct development in zebrafish [35]. This report also showed that the knockdown of PCP family members led to sidedness defects somewhat similar to those seen in patients with syndromic BA. Interestingly, Garnaas et al. [36 $]$ observed a similar phenotype in zebrafish in which the retinoic acid receptor gamma $\mathrm{b}(\mathrm{rargb})$ is targeted by morpholino antisense oligonucleotides. These models suggest the involvement of other pathways in biliary development and organ sidedness, thus possibly also being involved in syndromic BA and perhaps more globally in BA in general.

While the connection between BA and cell polarity is not completely clear, recent reports have shown that mice with haploinsufficiency of sox 17 demonstrate loss of cholangiocyte polarity that results in shedding of cells into the duct lumen and consequent obstruction [37]. Such a scenario in BA patients, in which loss of cell polarity leads to accumulation of cell debris and a resulting obstruction, would be consistent with several of the above features noted in zebrafish models and in patients. Demonstration of abnormalities in Hedgehog signaling or in other inflammatory pathways in the zebrafish polarity models would further support such a model. Clear extrahepatic defects such as obstruction of the extrahepatic ducts and/or gallbladder would also strengthen the case for an importance of polarity pathways in BA pathogenesis.

Very recently, an intriguing zebrafish model of BA has managed to replicate the extrahepatic defects. Over the past several decades, droughts in New South Wales have been associated with outbreaks of a BA-like illness in lambs and calves, thought to be a result of their mothers grazing on Dysphania species [38]. Investigators utilized zebrafish to screen extracted fractions of Dysphania for compounds that resulted in biliary defects and eventually isolated a single active compound that when given to zebrafish results in extrahepatic defects. Interestingly, administration of this compound to cultured cholangiocytes results in defects in primary cilia, and treatment of cholangiocyte spheroids results in marked loss of epithelial polarity, suggesting that the compound may affect cell polarity (Pack et al. personal communication). These studies further support a potential importance of polarity pathways in mediating biliary defects in zebrafish and thus possibly mediating BA pathogenesis; further studies of the targets of this compound could yield valuable clues regarding BA.

The above models approximate BA in zebrafish. No model has yet been able to replicate all of the features of BA, such as extrahepatic atresia, intrahepatic duct proliferation, increased fibrosis, and association with laterality defects. Several of the models get some or most of these features, though, and clearly we have learned and will probably continue to learn about disease pathogenesis from these models. The models have uncovered mechanisms by which important pathways, such as cell polarity, Hedgehog signaling, and inflammatory pathways may be influenced by genetic or epigenetic changes, resulting in abnormalities in the biliary tree, inflammation, and fibrogenesis (Fig. 1). As mentioned above, one of the great strengths of zebrafish is the ability to construct multiple models, and the combination of all of the above models may uncover additional facets of disease pathogenesis.

\section{Models of Other Cholestatic Diseases in Zebrafish}

Because none of the BA models perfectly recapitulates the human disease, it seems somewhat arbitrary to divide models of cholestasis in zebrafish into BA and non-BA categories. The models discussed in this section, though, were not intended to model BA. Nevertheless, these animal models can inform our understanding not only of the intended diseases, but for disease mechanisms of cholestasis in general. This may in turn allow us to increase our understanding of any cholestatic disease, including BA.

One of the first cholestatic diseases to be modeled in zebrafish was Alagille syndrome, which in patients is characterized by bile duct paucity, cardiac defects, vertebral defects, facial dysmorphism, and other anomalies [6]. Discovery of the causative gene led to recognition of the Jagged/Notch pathway as an important mediator of biliary development $[39,40]$. Morpholino-mediated knockdown of Jagged and Notch orthologs in zebrafish led to establishment of an Alagille syndrome phenocopy, supporting the importance of this pathway in biliary development in zebrafish [41]. More recent studies on Jagged/Notch signaling in zebrafish biliary development have shown that there is continued importance of Notch signaling during biliary remodeling [42], similar to mammalian biliary remodeling [39, 40]. These studies support further use of zebrafish to model Alagille syndrome and to study the role of jagged/notch signaling.

Another early model of cholestatic disease was uncovered in a mutagenesis screen for hepatomegaly. While the underlying causes of hepatomegaly in this screen varied, of relevance to cholestasis was a model of choledochal cyst formation in $n f 2$ (merlin) mutants [43]. Choledochal cysts are cysts involving the bile ducts, typically the extrahepatic ducts, that often present as cholestasis and can appear in infancy [1]. There is occasionally overlap between BA and the presence of a choledochal cyst [44]. The $n f 2$ mutant remains one of the only genetic models of extrahepatic defects in zebrafish. Work on this pathway in other systems has shown that Nf2 is an important part of the Hippo pathway, regulating growth and organ size [45]. Further studies on $n f 2$ in zebrafish could help understand the importance of this pathway in extrahepatic biliary 


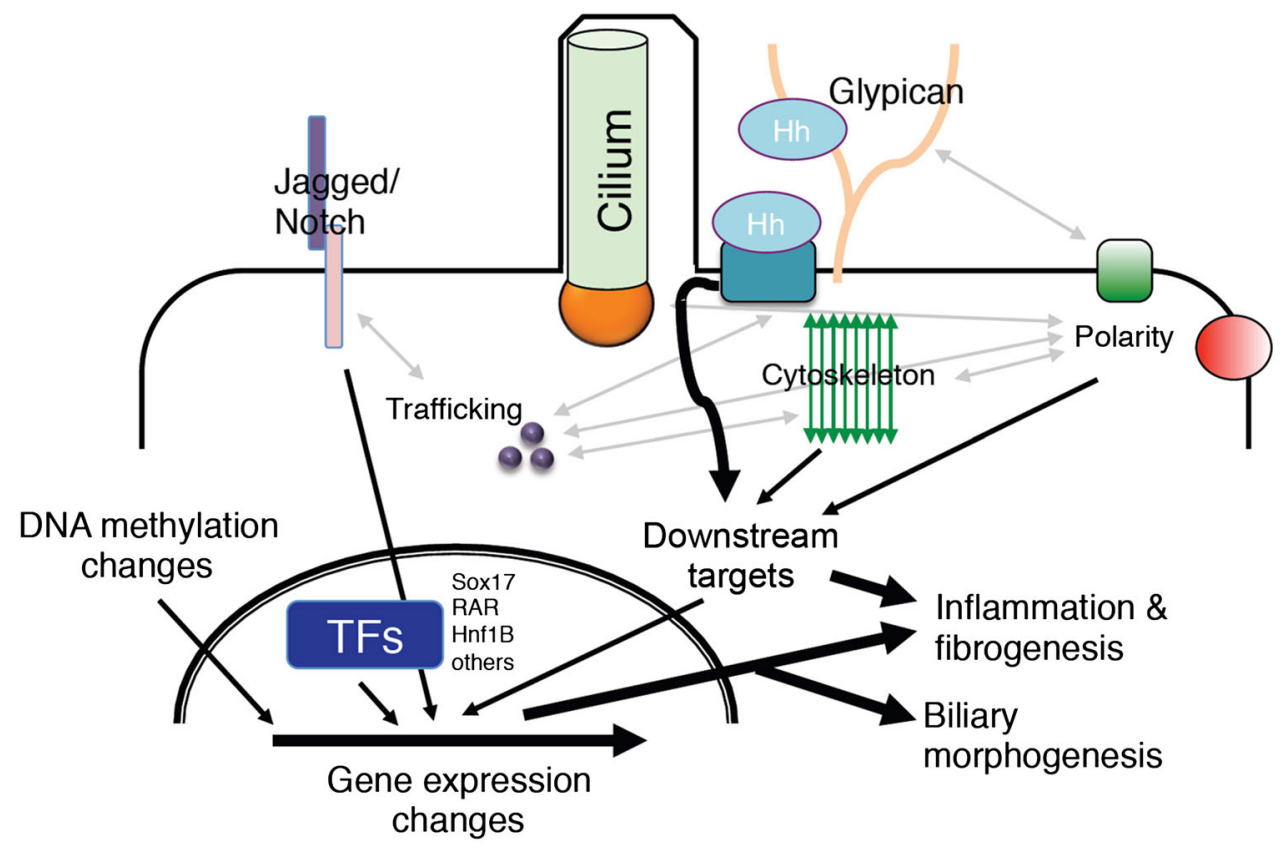

Fig. 1 Pathways implicated by studies on zebrafish models of pediatric cholestatic diseases. A schematic diagram depicting the pathways important in biliary development and in mediating biliary defects uncovered in zebrafish models of cholestatic diseases. Shown are pathways such as Jagged/Notch, primary cilia with Hedgehog signaling, polarity pathways, intracellular trafficking, and the cytoskeleton. The effects of these pathways on nuclear and other targets are noted by the dark arrows, while the interrelationships between these pathways are depicted by the gray arrows. Nuclear targets are mediated by these pathways and are depicted, or are affected by changes in DNA methylation, also noted

trafficking in biliary disease, as trafficking plays an important role in mediating cell polarity.

Central to the importance of cell polarity and primary cilia in biliary defects is increasing understanding of the mechanisms by which ciliopathies result in liver disease. The ciliopathies are a broad category of diseases, caused by genes important in cilia formation, in which patients have a characteristic set of findings that include liver and kidney cysts, brain abnormalities, and sidedness defects [10]. Liver defects can also include the ductal plate malformation, thought to be an arrest of biliary development, and relatively rapid progression of liver fibrosis [51]. While understanding the pathogenesis of these disorders is of itself important, the overlap between these disorders and some features of BA is intriguing and suggests some possible shared mechanisms. Several years ago, we determined that biliary development was abnormal in vhnfl (hnflb) mutants [52], in which there are also kidney cysts [53]. vhnfl, or Hnflb, is not generally considered a ciliopathy gene, however, as it encodes a transcription factor important in hepatobiliary development [54]. More recently, we have examined other kidney cyst mutants, and there are severe abnormalities in biliary development as well as activation of fibrogenesis genes (Cui and Matthews unpublished data). Others have shown 
conservation of these pathways in mediating liver cysts as well, as morpholino-mediated knockdown of several ciliopathy genes leads to liver cyst formation [55•]. These investigators stressed the utility of these models in future drug screening studies, which could also of course increase our understanding of the pathogenesis of these disorders as well.

Clear genetic causes of cholestasis and biliary defects in infants include Alagille syndrome, ARC syndrome, ciliopathies, and North American Indian childhood cirrhosis (NAIC). NAIC is a condition of particular interest, in that in patients it is indistinguishable from BA in terms of symptoms and the intrahepatic defects, but without extrahepatic defects $[8,56]$. Recently, a zebrafish model of NAIC was reported, utilizing morpholinos to knockdown the causative gene, cirhla [57]. The intrahepatic defects seen in this model appeared to be mediated by p53-mediated cell death, suggesting a potential importance of this pathway in NAIC.

The above models have established the utility of zebrafish in studying genetic causes of cholestasis. There is remarkable conservation of the importance of these molecular pathways. Further study of these pathways in the zebrafish models should increase our understanding of the human disorders and may help uncover treatments for these disorders as well.

\section{Models of Acute Liver Failure and Metabolic Disease}

To date, most models of acute liver failure in zebrafish have been generated as tools to study liver regeneration, but these models could serve to examine the disease process as well. There is hepatocyte death in larvae with homozygous mutation of tomm22, which encodes a mitochondrial import gene [58], and in larvae engineered to overexpress the nitroreductase enzyme in the liver, which leads to hepatocyte death after treatment with metronidazole [59]. Use of other toxins, such as the well-studied liver toxin acetaminophen, also leads to hepatocyte death in larval zebrafish, and studies have uncovered potentially novel pathways and treatments for reversing the effects of acetaminophen [60].

The mutant duct-trip, described above, demonstrates an ALF phenotype in addition to the biliary defects. This phenotype is not elicited by inhibition of DNA methylation and is rescued by inhibition of TNF $\alpha$ [16]. The ALF phenotype in duct-trip was associated with mitochondrial

Table 1 Summary of models described in this review, showing manipulated gene or toxin, phenotype, pathway, and method

\begin{tabular}{|c|c|c|c|c|}
\hline Gene & Disease/phenotype & Pathway/target & Method & References \\
\hline Ahcy & Biliary atresia & Methylation & Mutation & {$\left[16,17^{\bullet}\right]$} \\
\hline dnmt1 & Biliary atresia & DNA methylation & Morpholino knockdown & {$[17 \bullet]$} \\
\hline azacytidine & Biliary atresia & DNA methylation & Chemical treatment & {$[17 \bullet, 18]$} \\
\hline$z f h x 1 b$ & Biliary atresia & SMAD signaling & Morpholino knockdown & {$[21]$} \\
\hline gpcl & Biliary atresia & Hedgehog & Morpholino knockdown & {$[22 \bullet \bullet]$} \\
\hline Claudin15bl & Biliary atresia & Polarity & Mutation & {$[34 \bullet]$} \\
\hline Prickle1a & Biliary atresia (syndromic) & Planar cell polarity & Morpholino knockdown & {$[35]$} \\
\hline Rargb & Biliary atresia (syndromic) & Polarity/Retinoic acid & Morpholino knockdown & {$[36 \bullet]$} \\
\hline Unknown & Biliary atresia & Unknown - cilia? & Dysphania exposure & Pers. comm. \\
\hline Jagged/Notch & Alagille syndrome & Notch & Morpholino knockdown & {$[41,42]$} \\
\hline$n f 2$ & Choledochal cyst & Hippo & Insertional mutagenesis & {$[43]$} \\
\hline vps33b & ARC syndrome & Intracellular trafficking & Morpholino knockdown & [46] \\
\hline vps 18 & Cholestasis/ARC-like & Intracellular trafficking & Insertional mutagenesis & {$[43]$} \\
\hline vps39 & Cholestasis/ARC-like & Intracellular trafficking & Mutation & {$[47]$} \\
\hline atp6ap2 & Cholestasis/ARC-like & Intracellular compartment $\mathrm{pH}$ & Mutation & {$[48 \bullet]$} \\
\hline$h n f 1 b$ & Ciliopathy-like & Hepatobiliary development & Insertional mutagenesis & {$[52]$} \\
\hline pkhd1 & Ciliopathy & Polarity/cilia & Morpholino knockdown & Unpub. data \\
\hline cirhla & North American Indian childhood cirrhosis & p53 & Morpholino knockdown & {$[57]$} \\
\hline heg1 & Cholestasis? & Apicobasolateral polarity (indirectly) & Mutation & {$[33]$} \\
\hline
\end{tabular}

References are provided 
abnormalities and fatty liver, and there is considerable overlap between ALF and hepatic steatosis in other zebrafish models [61] and in patients, particularly those with ALF secondary to mitochondrial disorders. Supporting this mechanism, we have elicited hepatic steatosis in larval zebrafish using the mitochondrial toxin valinomycin (Sapp et al. submitted). These models, similar to those described above, have not been used directly to examine ALF, but there would seem to be an opportunity here to address the pathogenesis of early ALF using the above models.

\section{Conclusions}

We have presented several models of infantile cholestatic and metabolic diseases in zebrafish (Table 1). The pathogenesis of many of these disorders, including the most common identifiable infantile cholestatic disorder, is not well understood, and zebrafish models offer an intriguing method to better understand these diseases. The models utilize frequently touted advantages of zebrafish, such as the ease of genetic manipulation, rapid development time, and the relative ease of establishing transgenic lines. Moreover, the existence of multiple models allows for the comparison of models to uncover similarities that may be important in the underlying pathogenic mechanisms.

This analysis has uncovered a potential importance of trafficking and polarity pathways in mediating biliary defects, which as detailed above is supported by work in mammalian models and in patients that show involvement of the pathways in disease. In addition to the importance of polarity pathways in cholestatic diseases such as BA, the zebrafish models have also uncovered a potential importance of DNA methylation in BA. Future studies, in zebrafish, mammalian models, and in patients, will help to solidify these findings and will of course continue to uncover interesting facets of disease pathogenesis.

Future studies on cholestatic disease using zebrafish, as with other models in zebrafish, will likely be tremendously exciting. New technologies that allow for more facile engineering of mutants, such as TALENs and CRISPR/ Cas, will allow us to examine the roles of multiple genes and pathways in zebrafish models. Continued close work with our geneticist colleagues will prove fruitful as well, as using the same technologies we can examine the potential importance of specific mutations and advance the nascent field of "personalized medicine." We can also take advantage of our multiple models to uncover possible treatments for these disorders. These continued translational efforts should lead to great advances in the understanding of pediatric cholestatic diseases.
Acknowledgments We thank Drs. Michael Pack, John Porter, and Rebecca Wells for sharing their data prior to publication. The authors are supported by NIH R01 DK090260.

\section{Compliance with Ethics Guidelines}

Conflict of Interest Randolph Matthews and Zenobia Cofer declare they have no conflict of interest.

Human and Animal Rights and Informed Consent This article does not contain any studies with human or animal subjects performed by any of the authors.

\section{References}

Papers of particular interest, published recently, have been highlighted as:

- Of importance

•. Of major importance

1. Suchy FJ (2004) Neonatal cholestasis. Pediatr Rev 25(11): 388-396

2. Haber BA, Russo P (2003) Biliary atresia. Gastroenterol Clin N Am 32(3):891-911

3. Davenport M et al (2006) The biliary atresia splenic malformation syndrome: a 28 -year single-center retrospective study. J Pediatr 149(3):393-400

4. Schwarz KB et al (2013) Extrahepatic anomalies in infants with biliary atresia: results of a large prospective North American multicenter study. Hepatology 58(5):1724-1731

5. Shneider BL et al (2006) A multicenter study of the outcome of biliary atresia in the United States, 1997 to 2000. J Pediatr 148(4):467-474

6. Piccoli DA, Spinner NB (2001) Alagille syndrome and the Jagged1 gene. Semin Liver Dis 21(4):525-534

7. Gissen P et al (2004) Mutations in VPS33B, encoding a regulator of SNARE-dependent membrane fusion, cause arthrogryposisrenal dysfunction-cholestasis (ARC) syndrome. Nat Genet 36(4):400-404

8. Drouin E et al (2000) North American Indian cirrhosis in children: a review of 30 cases. J Pediatr Gastroenterol Nutr 31(4): 395-404

9. Kerkar N, Norton K, Suchy FJ (2006) The hepatic fibrocystic diseases. Clin Liver Dis 10(1):55-71 v-vi

10. Hildebrandt F, Benzing T, Katsanis N (2011) Ciliopathies. N Engl J Med 364(16):1533-1543

11. Sun Zet al (2004) A genetic screen in zebrafish identifies cilia genes as a principal cause of cystic kidney. Development 131(16): 4085-4093

12. Sullivan-Brown $\mathrm{J}$ et al (2008) Zebrafish mutations affecting cilia motility share similar cystic phenotypes and suggest a mechanism of cyst formation that differs from pkd2 morphants. Dev Biol 314(2):261-275

13. Squires RH Jr et al (2006) Acute liver failure in children: the first 348 patients in the pediatric acute liver failure study group. J Pediatr 148(5):652-658

14. Vajro P et al (2012) Diagnosis of nonalcoholic fatty liver disease in children and adolescents: position paper of the ESPGHAN Hepatology Committee. J Pediatr Gastroenterol Nutr 54(5): 700-713 
15. Petersen C et al (1997) New aspects in a murine model for extrahepatic biliary atresia. J Pediatr Surg 32(8):1190-1195

16. Matthews RP et al (2009) TNF\{alpha\}-dependent hepatic steatosis and liver degeneration caused by mutation of zebrafish $s$ adenosylhomocysteine hydrolase. Development 136(5):865-875

17. - Matthews RP et al (2011) DNA hypomethylation causes bile duct defects in zebrafish and is a distinguishing feature of infantile biliary atresia. Hepatology. PMID 21319190. Using zebrafish models derived genetically, by drug treatment, and by morpholino injection, the authors show that inhibition of DNA methylation leads to biliary defects. They also show that there is decreased DNA methylation in bile ducts of patients with biliary atresia

18. Cui S, Eauclaire SF, Matthews RP (2013) Interferon-gamma directly mediates developmental biliary defects. Zebrafish 10:177-183

19. Bezerra JA et al (2002) Genetic induction of proinflammatory immunity in children with biliary atresia. Lancet 360(9346): $1653-1659$

20. Shivakumar P et al (2004) Obstruction of extrahepatic bile ducts by lymphocytes is regulated by IFN-gamma in experimental biliary atresia. J Clin Investig 114(3):322-329

21. Cui $S$ et al (2011) Intrahepatic biliary anomalies in a patient with Mowat-Wilson syndrome uncover a role for the zinc finger homeobox gene zfhx $1 \mathrm{~b}$ in vertebrate biliary development. J Pediatr Gastroenterol Nutr 52(3):339-344

22. • Cui S et al (2013) Evidence from human and zebrafish that GPC1 is a biliary atresia susceptibility gene. Gastroenterology. 144:1107-1115. PMID 23336978. The authors identify GPC1 as a potential biliary atresia risk gene and use zebrafish to show that gpcl knockdown leads to biliary defects and activation of Hedgehog, similar to biliary atresia

23. Leyva-Vega M et al (2010) Genomic alterations in biliary atresia suggest region of potential disease susceptibility in 2q37.3. Am J Med Genet A 152A(4):886-895

24. Tsai EA et al (2013) Replication of a GWAS signal in a Caucasian population implicates ADD3 in susceptibility to biliary atresia. Hum Genet 133(2):235-243

25. Garcia-Barcelo MM et al (2010) Genome-wide association study identifies a susceptibility locus for biliary atresia on 10q24.2. Hum Mol Genet 19(14):2917-2925

26. Yan D, Lin X (2008) Opposing roles for glypicans in Hedgehog signalling. Nat Cell Biol 10(7):761-763

27. Omenetti A et al (2011) Hedgehog activity, epithelial-mesenchymal transitions, and biliary dysmorphogenesis in biliary atresia. Hepatology 53(4):1246-1258

28. Chu AS, Russo PA, Wells RG (2012) Cholangiocyte cilia are abnormal in syndromic and non-syndromic biliary atresia. Mod Pathol 25(5):751-757

29. • Yin C et al (2013) Hepatic stellate cells in liver development, regeneration, and cancer. J Clin Investig 123(5):1902-1910. PMID 23635788. The authors present a novel transgenic fish in which hepatic stellate cells are labeled, allowing these cells to be followed during development and in models of disease states

30. D'Angelo A, Franco B (2009) The dynamic cilium in human diseases. Pathogenetics 2(1):3

31. Park TJ, Haigo SL, Wallingford JB (2006) Ciliogenesis defects in embryos lacking inturned or fuzzy function are associated with failure of planar cell polarity and Hedgehog signaling. Nat Genet 38(3):303-311

32. Desbordes SC, Sanson B (2003) The glypican Dally-like is required for Hedgehog signalling in the embryonic epidermis of Drosophila. Development 130(25):6245-6255

33. Sakaguchi TF et al (2008) Endothelial signals modulate hepatocyte apicobasal polarization in zebrafish. Curr Biol 18(20): 1565-1571
34. - Cheung ID et al (2011) Regulation of intrahepatic biliary duct morphogenesis by Claudin 15-like b. Dev Biol 361(1):68-78. PMID 22020048. The authors show that a gene important in mediating hepatocyte polarity has a role in intrahepatic biliary development

35. Cui S, Capecci LM, Matthews RP (2011) Disruption of planar cell polarity activity leads to developmental biliary defects. Dev Biol 351(2):229-241

36. • Garnaas MK et al (2012) Rargb regulates organ laterality in a zebrafish model of right atrial isomerism. Dev Biol 372(2):178-89. PMID 22982668. The authors show that manipulation of the retinoic acid signaling pathway leads to biliary defects and to left-right asymmetry defects

37. Uemura $M$ et al (2013) Sox17 haploinsufficiency results in perinatal biliary atresia and hepatitis in C57BL/6 background mice. Development 140(3):639-648

38. Harper P, Plant JW, Unger DB (1990) Congenital biliary atresia and jaundice in lambs and calves. Aust Vet J 67(1):18-22

39. Kodama $\mathrm{Y}$ et al (2004) The role of notch signaling in the development of intrahepatic bile ducts. Gastroenterology 127(6): $1775-1786$

40. Zong $\mathrm{Y}$ et al (2009) Notch signaling controls liver development by regulating biliary differentiation. Development 136(10): $1727-1739$

41. Lorent $\mathrm{K}$ et al (2004) Inhibition of Jagged-mediated Notch signaling disrupts zebrafish biliary development and generates multi-organ defects compatible with an Alagille syndrome phenocopy. Development 131(22):5753-5766

42. Lorent $\mathrm{K}$ et al (2010) Reiterative use of the notch signal during zebrafish intrahepatic biliary development. Dev Dyn 239(3): 855-864

43. Sadler KC et al (2005) A genetic screen in zebrafish identifies the mutants vps18, nf2 and foie gras as models of liver disease. Development 132(15):3561-3572

44. Muise AM et al (2006) Biliary atresia with choledochal cyst: implications for classification. Clin Gastroenterol Hepatol 4(11): $1411-1414$

45. Stamenkovic I, Yu Q (2010) Merlin, a "magic" linker between extracellular cues and intracellular signaling pathways that regulate cell motility, proliferation, and survival. Curr Protein Pept Sci 11(6):471-484

46. Matthews RP et al (2005) Zebrafish vps33b, an ortholog of the gene responsible for human arthrogryposis-renal dysfunctioncholestasis syndrome, regulates biliary development downstream of the onecut transcription factor hnf6. Development 132(23): 5295-5306

47. Schonthaler HB et al (2008) The zebrafish mutant lbk/vam6 resembles human multisystemic disorders caused by aberrant trafficking of endosomal vesicles. Development 135(2):387-399

48. - Eauclaire SF et al (2012) Mutations in vacuolar H(+)-ATPase subunits lead to biliary developmental defects in zebrafish. Dev Biol. PMID 22465374. The authors describe biliary mutants, including a biliary mutant caused by mutation of a gene important in mediating intracellular compartment $\mathrm{pH}$

49. Forgac M (1998) Structure, function and regulation of the vacuolar (H+)-ATPases. FEBS Lett 440(3):258-263

50. Cullinane AR et al (2010) Mutations in VIPAR cause an arthrogryposis, renal dysfunction and cholestasis syndrome phenotype with defects in epithelial polarization. Nat Genet 42(4): 303-312

51. Roskams T, Desmet V (2008) Embryology of extra- and intrahepatic bile ducts, the ductal plate. Anat Rec (Hoboken) 291(6): 628-635

52. Matthews RP et al (2004) The zebrafish onecut gene hnf-6 functions in an evolutionarily conserved genetic pathway that regulates vertebrate biliary development. Dev Biol 274(2):245-259 
53. Sun Z, Hopkins N (2001) vhnf1, the MODY5 and familial GCKD-associated gene, regulates regional specification of the zebrafish gut, pronephros, and hindbrain. Genes Dev 15(23): 3217-3229

54. Coffinier C et al (2002) Bile system morphogenesis defects and liver dysfunction upon targeted deletion of HNF1beta. Development 129(8):1829-1838

55. - Tietz Bogert PS et al (2013) The zebrafish as a model to study polycystic liver disease. Zebrafish 10(2):211-217. PMID 23668934. The authors describe models of polycystic liver and kidney disease, and show proof-of-principle in use of the models to find potential drug treatments for these defects

56. Chagnon $\mathrm{P}$ et al (2002) A missense mutation (R565W) in cirhin (FLJ14728) in North American Indian childhood cirrhosis. Am J Hum Genet 71(6):1443-1449

57. Wilkins BJ et al (2013) p53-Mediated biliary defects caused by knockdown of cirhla, the zebrafish homolog of the gene responsible for North American Indian Childhood Cirrhosis. PLoS ONE 8(10):e77670

58. Curado $S$ et al (2010) The mitochondrial import gene tomm22 is specifically required for hepatocyte survival and provides a liver regeneration model. Dis Model Mech 3(7-8):486-495

59. Curado S, Stainier DY, Anderson RM (2008) Nitroreductasemediated cell/tissue ablation in zebrafish: a spatially and temporally controlled ablation method with applications in developmental and regeneration studies. Nat Protoc 3(6):948-954

60. North TE et al (2010) PGE2-regulated wnt signaling and $N$ acetylcysteine are synergistically hepatoprotective in zebrafish acetaminophen injury. Proc Natl Acad Sci USA 107(40): $17315-17320$

61. Howarth DL et al (2013) Defining hepatic dysfunction parameters in two models of fatty liver disease in zebrafish larvae. Zebrafish 10(2):199-210 